\title{
Mean platelet volume as a diagnostic parameter of respiratory syncytial virus infection
}

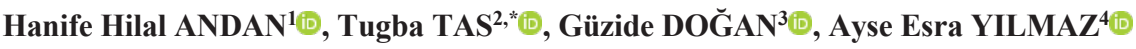

${ }^{1}$ Department of Pediatrics, Faculty of Medicine, Turgut Ozal University, Ankara, Turkey

${ }^{2}$ Department of Pediatrics, Hisar Intercontinental Hospital, Istanbul, Turkey

${ }^{3}$ Department of Pediatrics, Department of Gastroenterology, Haseki Research and Training Hospital, Istanbul, Turkey

${ }^{4}$ Department of Pediatrics, Medical Park Hospital, Ankara, Turkey

\begin{tabular}{ccccc}
\hline Received: 14.12 .2020 & $\bullet$ & Accepted/Published Online: 21.03 .2021 & $\bullet$ & Final Version: 23.04 .2021 \\
\hline
\end{tabular}

\begin{abstract}
Our aim was to investigate RSV related parameters by comparing clinical findings and physical examination with routinely ordered whole blood counts and biochemical variables in under two age children hospitalized for lower respiratory tract infections. The sample consisted of 193 children [RSV positive $(n=85)$, RSV negative $(n=108)$ ] with lower respiratory tract infections from May 2010 to May 2013. Sociodemographic findings, chief complaints and physical examination findings were retrospectively evaluated. RSV positive and negative groups were compared using Chi-square test ( $\% 95$ Confidence Interval- $\% 95 \mathrm{CI}$ ), Mann-Whitney $U$ test $(\mathrm{p}=0.05)$. No significant differences were found in demographic variables and treatment decisions between two groups. In RSV positive group, higher hyponatremia $(\mathrm{p} \leq 0.001)$ and AST values ( $\mathrm{p}=0.003)$ were found. Decreased MPV were determined 71.8\% in RSV positive group. RSV positive patients had decreased MPV values (OR:10.929, 95\% CI). Higher hyponatremia and increased AST values were found to be associated with RSV infection; and decreased MPV was significantly related with RSV infection. This is the second study in literature within our knowledge that found decreased MPV values in RSV positive patients. The mechanism related with decreased MPV, hyponatremia, and increased AST values in RSV infection should be investigated further.
\end{abstract}

Keywords: RSV, mean platelet volume, bronchiolitis, infection

\section{Introduction}

According to data from World Health organization (WHO), three million of infants and children die because of lower respiratory tract infections (LRTI) annually. Respiratory Syncytial Virus (RSV) is the most important causative agent of viral LRTIs in infants and children all around the world. The widely use of serologic tests in recent years for diagnosis have shown that RSV infection may occur in any climate and geographic region (Murray et al., 1999). RSV infection is most commonly seen in children under two years of age. Almost half of the children who have infection just involving upper respiratory tract, experienced a progression of infection to lower respiratory tract. Hospitalization rate is about $2 \%$. Mortality rate in hospitalized patients is $0.5-1 \%$ (La Vla et al., 1992; Collins and Chanock, 1996).

Children with a history of prematurity, bronchopulmonary dysplasia, congenital or acquired immune deficiency and haematological malignancies, bone marrow or organ transplantation, cystic fibrosis, oxygen supply at home, neurologic and metabolic diseases are all at higher risk for having RSV infection. The disease is prolonged and causes higher mortality in children with congenital immune deficiency (Walsh et al., 1997; Handforth et al., 2000). The mortality rate is higher in children with congenital heart disease those have already impaired respiratory and cardiac functions. Prognosis worsens in the presence of pulmonary hypertension (Geskey and Cyran, 2012). Almost all infants and children have RSV infection within first two years of life. Reinfections can be seen because of immature host defense (Ohuma et al., 2012). RSV infection is a major public health problem due to high morbidity and mortality in young children and a rapid diagnosis and treatment should be made to prevent spread of disease. Educating health care providers is also an important key point (Prober and Sullender, 1999).

Diagnosis of RSV infection can be made by different tests including isolation of the virus from cell culture or detection of antigens by serologic and immunochromatographic methods. Detection of RSV antigen is made through directly from nasopharyngeal swab, nasopharyngeal aspirate or nasal / nasopharyngeal wash specimens. Nasopharyngeal aspirate method is referred as gold standard method in many literature to detect RSV antigen. Laboratory diagnosis of RSV infection is important for public health and health economics. Rapid 
diagnosis avoids unnecessary use of antibiotics, nosocomial spread and provides early beginning of antiviral treatment for children with serious illness (Luksic et al., 2013). Although rapid diagnosis of RSV is important for prompt treatment, there are no appropriate testing procedures in most of health institutions of our country and many developing countries.

In this study, we aimed to compare clinical findings, complete blood count and biochemical parameters of 1-24 month-old infants, who treated in outpatient clinic or hospitalized for RSV (+) or RSV (-) viral LRTI for setting RSV identifier parameters.

\section{Materials and methods}

A total of 193 infants aged 1- to 24-month-old were enrolled for study between May 2010 and May 2013 in Turgut Ozal University Faculty of Medicine Hospital with clinical findings of viral LRTI. All patient were tested for RSV antigen by immunochromatographic method. Infants with severe illnesses (sepsis, meningitis), serious neurological and metabolic disorders, immune deficiencies, and suspected bacterial infections were excluded from study.

Demographic data of patients fulfilling the criteria were collected through a computer program. Lacking information was completed by phone calls with families. Detailed history of patients including age, gender, chief complaints, beginning time of the symptoms, number of siblings, presence of sibling ongoing day care or school, comorbidity, smoke exposure, apnea, prematurity and birth season were recorded.

Hospitalization, oxygen requirement, need for antibiotic and other treatment options were extracted from study forms. The patients were followed up in terms of recurrent wheezing through records for a 6 months period after enrollment. Signs and symptoms, laboratory investigations, radiologic findings were recorded. All patients underwent rapid RSV immunochromatographic testing with samples obtained by nasopharyngeal swabs. Patients were assigned into two groups according to recorded test results in study form as RSV antigen positive and negative.

\subsection{Statistical analysis}

The normal distribution variables were evaluated using Shapiro- Wilk test. Skewed variables were expressed as median (Interquantile ranges). Counts and percentages were used for categorical variables. Comparison of data based on differences between study groups and difference in proportions were analyzed by performing Chi-square test and Likelihood ratio. Crude and adjusted odds ratios (OR) were obtained with $95 \%$ confidence intervals (95\% CI). Correlation analyses were performed with Spearman Rho correlation coefficient test for variables with nonparametric distribution. The Mann Whitney U-test was used to compare the two groups. All statistical analyses were performed using IBM SPSS Statistics 21.0 (IBM

Corp. Released 2012. IBM SPSS Statistics for Windows, Version 21.0. Armonk, NY: IBM Corp.) and MS-Excel 2007.
Significance was assigned at $\mathrm{p}<0.05$.

Informed consent for participation in this study was obtained from the patients' parents or guardians. The study protocol was approved by the Ethics Committee of the Turgut Ozal University, Faculty of Medicine.

\section{Results}

The study group consisted of 183 children, 79 females (40.9\%) and 114 males (59.1\%). There were 151 infants (78.2\%) under the age of one, and 42 toddlers $(21.8 \%)$ between ages 1 and 2 . The median gestational age was 39 weeks. Prematurity rate was $11.9 \%(n=23)$. Forty-nine patients $(25.4 \%)$ had smoke exposure story. Positivity for RSV was $44 \%$, and did not show statistically significant difference with respect to gender. Patients with a history of premature birth rate did not significantly differ for both groups. That is, no significant difference was detected between RSV positivity and all demographic features shown as Table 1 ( $\mathrm{p}>0.05)$.

Table 1. Comparison of demographic features according to rsv positivity

\begin{tabular}{|c|c|c|c|c|}
\hline & $\begin{array}{c}\text { Negative } \\
\mathbf{n}(\%)\end{array}$ & $\begin{array}{c}\text { Positive } \\
\text { n(\%) }\end{array}$ & $\chi^{2}$ & $\mathbf{P}$ \\
\hline \multicolumn{5}{|l|}{ Gender } \\
\hline $\begin{array}{l}\text { Female } \\
\text { Male }\end{array}$ & $\begin{array}{l}41(51.9) \\
67(58.8)\end{array}$ & $\begin{array}{l}38(48.1) \\
47(41.2)\end{array}$ & 0.894 & 40.344 \\
\hline \multicolumn{5}{|l|}{ Age } \\
\hline $\begin{array}{l}<12 \text { month } \\
12-24 \text { month }\end{array}$ & $\begin{array}{l}81(53.6) \\
27(64.3)\end{array}$ & $\begin{array}{l}70(46.4) \\
15(35.7)\end{array}$ & 1.510 & 0.219 \\
\hline \multicolumn{5}{|c|}{ Smoke exposure } \\
\hline $\begin{array}{l}\text { No } \\
\text { Yes }\end{array}$ & $\begin{array}{l}79(54.9) \\
29(59.2)\end{array}$ & $\begin{array}{l}65(45.1) \\
20(40.8)\end{array}$ & 0.277 & 70.599 \\
\hline \multicolumn{5}{|l|}{ Prematurity } \\
\hline $\begin{array}{l}\text { No } \\
\text { Yes }\end{array}$ & $\begin{array}{c}99(58.2) \\
9(39.1)\end{array}$ & $\begin{array}{l}71(41.8) \\
14(60.9)\end{array}$ & 3.000 & 0.083 \\
\hline \multicolumn{5}{|l|}{$\begin{array}{l}\text { Sibling at day } \\
\text { care }\end{array}$} \\
\hline $\begin{array}{l}\text { No } \\
\text { Yes }\end{array}$ & $\begin{array}{l}56(52.8) \\
52(59.8)\end{array}$ & $\begin{array}{l}50(47.2) \\
35(40.2)\end{array}$ & 0.934 & 40.334 \\
\hline \multicolumn{5}{|l|}{ Breastfeeding } \\
\hline $\begin{array}{l}\text { No } \\
\text { Yes }\end{array}$ & $\begin{array}{l}27(62.8) \\
81(54.0)\end{array}$ & $\begin{array}{l}16(37.2) \\
69(46.0)\end{array}$ & 1.048 & 0.306 \\
\hline \multicolumn{5}{|c|}{ Hospitalization } \\
\hline $\begin{array}{l}\text { No } \\
\text { Yes }\end{array}$ & $\begin{array}{l}20(45.5) \\
88(59.1)\end{array}$ & $\begin{array}{l}24(54.5) \\
61(40.9)\end{array}$ & 2.552 & 20.110 \\
\hline
\end{tabular}

The median hospitalization duration was $3.5(\mathrm{IR}=3)$ in RSV (-) patients, and $4.0(\operatorname{IR}=2)$ in $\mathrm{RSV}(+)$ patients $(Z=1.878 ; \quad p=0.060)$. Need for oxygen, oxygen supplementation duration and gestation week did not significantly differ between groups. Signs and symptoms such as fever, tachypnea, retraction and existence of apnea were also similar for RSV (+) and RSV (-) patients. While the number of patients who have oxygen saturation level below $92 \%$ was 45 $(52.9 \%)$ for RSV (+) group, it was 60 (55.6\%) for RSV () patients. Recurrent wheezing episode rate in follow-up period was $28.2 \%(n=24)$ for RSV $(+)$ group and $31.5 \%(n=34)$ for RSV (-). 
Table 2. The distribution of treatment practices based on RSV positivity and negativity

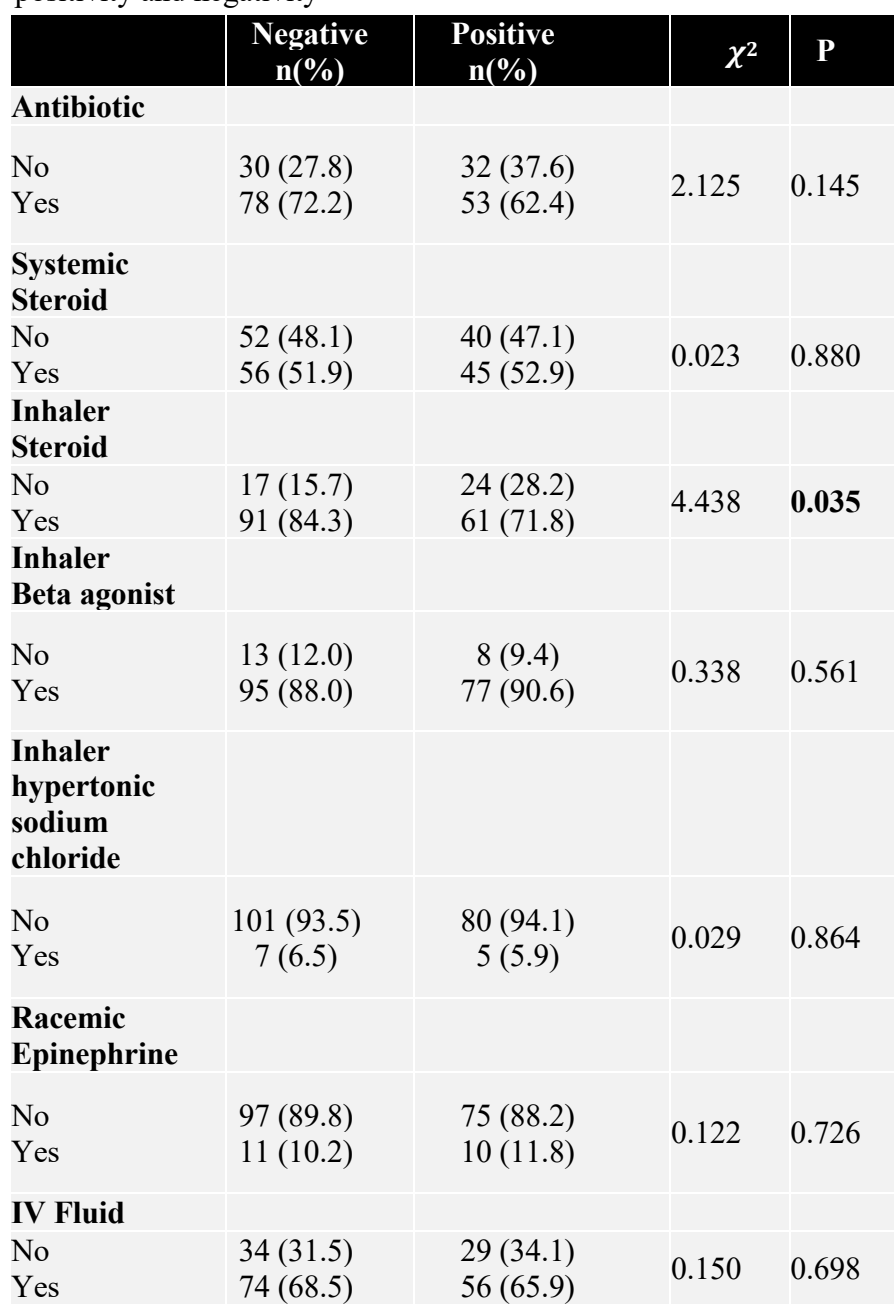

Although all patients had a LRTI, antibiotic use was very high in both study groups $(62.4 \%$ in RSV (+) group, $72.2 \%$ in RSV (-) group). RSV positivity had no effect on systemic steroid or inhaler beta agonist use $(\mathrm{p}=0.561)$. Similarly; inhaler hypertonic sodium chloride, racemic epinephrine use and intravenous fluid administration did not differ significantly between groups ( $\mathrm{p}>0.05)$. But interestingly, inhaler steroid use rate was 84,3 \% (n 91) in RSV (-) group (Table 2). Four patients with RSV bronchiolitis and three from other group developed heart failure and received digoxin. White blood cell and platelet count of 106 patients did not show significantly difference whether the patient is $\operatorname{RSV}(+)$ or not $(\mathrm{p}=0.541)$. We evaluated serum aspartate aminotransferase (AST), alanine aminotransferase (ALT) and sodium (Na) levels of 103 patients. Despite serum AST level was higher in RSV (+) group ( $\mathrm{p}=0.003$ ), ALT level was not different. In about of 31.7 $\%$ RSV (+) group serum sodium $\mathrm{Na}$ ) level was significantly low in $(\mathrm{p}<0.001)$, additionally none of group members had increased urine specific gravity in contrast to RSV (-) group $(\mathrm{p}=0.007)$ (Table 3$)$. Findings in chest $\mathrm{x}$-ray and C-reactive protein (CRP) levels were similar for both groups ( $p>0.05)$. RSV infection was more frequent in infants those born in winter and early $(\mathrm{p}=0.008)$.
Table 3. The distribution laboratory investigations based on RSV positivity and negativity

\begin{tabular}{|c|c|c|c|c|}
\hline Normal Ranges & $\begin{array}{c}\text { Negative } \\
\text { n(\%) }\end{array}$ & $\begin{array}{c}\text { Positivie } \\
\text { n(\%) }\end{array}$ & $\chi^{2}$ & $\mathbf{P}$ \\
\hline MPV & & & \multirow[b]{2}{*}{54.043} & \multirow[b]{2}{*}{$<0.001$} \\
\hline $\begin{array}{l}(7.8-11.1) \text { n }(\%) \\
\text { Low }(<7.8) \text { n }(\%)\end{array}$ & $\begin{array}{l}86(81.1) \\
20(18.9)\end{array}$ & $\begin{array}{l}24(28.2) \\
61(71.8)\end{array}$ & & \\
\hline AST & & & \multirow[b]{2}{*}{8.97} & \multirow[b]{2}{*}{0.003} \\
\hline $\begin{array}{l}(\leq 40 \mathrm{U} / \mathrm{L}) \\
\operatorname{High}(>40 \mathrm{U} / \mathrm{L})\end{array}$ & $\begin{array}{l}78(75.7) \\
25(24.3)\end{array}$ & $\begin{array}{l}45(54.9) \\
37(45.1)\end{array}$ & & \\
\hline $\mathbf{N a}$ & & & \multirow[b]{2}{*}{21.379} & \multirow[b]{2}{*}{$<0.001$} \\
\hline $\begin{array}{l}(>135 \mathrm{mg} / \mathrm{dL}) \\
\text { Low }(<135 \mathrm{mg} / \mathrm{dL}) \\
\text { Urine Specific } \\
\text { Gravity }\end{array}$ & $\begin{array}{c}97(94.2) \\
6(5.8)\end{array}$ & $\begin{array}{l}56(68.3) \\
26(31.7)\end{array}$ & & \\
\hline $\begin{array}{l}(1005-1020) \\
\text { High }(>1020)\end{array}$ & $\begin{array}{c}48(87.3) \\
7(12.7)\end{array}$ & $\begin{array}{c}35(100) \\
0(0.0)\end{array}$ & 7.267 & $0.007 * *$ \\
\hline
\end{tabular}

There was a significant association between decreased mean platelet volume (MPV) and RSV infection as shown on Table $3(\mathrm{p}<0.001)$. None of study patients had MPV value above limits. Decreased MPV rate was higher in RSV (+) group than RSV (-) group (71.8\% and $18.9 \%$ respectively). The patients with RSV $(+)$ lower respiratory tract infection were about 10 times more prone to have decreased MPV level $(\mathrm{OR}=10.929 ; \% 95 \mathrm{CI})$. Oxygen saturation and duration of oxygen supply were not related with low hemoglobin level $(\mathrm{p}=0.507$ and $\mathrm{p}=0.590$ respectively). The median mean corpuscular volume (MCV) and mean corpuscular hemoglobin concentration (MCHC) values in $\mathrm{RSV}(+)$ group were significantly higher than RSV (-) group $(\mathrm{p}=0.003)$. RDW median was similar in both groups $(\mathrm{p}=0.276)$. Of the twentythree patients with premature birth story, three infants (17.6\%) had received palivizumab, 5 (\%21.7) had needed oxygen supplementation. Palivizumab therapy had no effect on median durations for hospitalization and oxygen need $(p=0953$ and $\mathrm{p}=0.801$ respectively). Of $60.0 \%$ premature infants those have received palivizumab for palivizumab and $77.8 \%$ who have not received were hospitalized. Data were not enough statistically to conclude about palivizumab effect for RSV infection prophylaxis.

\section{Discussion}

Acute bronchiolitis is a common LRTI in childhood. Males are more prone to bronchiolitis caused by RSV or other etiologic factors (Boyce et al., 2000; Kristensen et al., 2009). We have observed that a higher proportion of bronchiolitis were in male gender and the male/female ratio was $1.3 / 1$. Since airways of male infants are narrower than female infants, bronchial inflammation caused by LRTI reduces the size of airways further. Their airways become more susceptible to infections (Boyce et al., 2000; Kristensen et al., 2009; Navas et al., 1999). Lamarao et al. conducted a study with 1050 hospitalized infants for acute bronchiolitis and showed that bronchiolitis rate was higher (\%55.6) in males (Lamarao et al., 2012). In our 
study, we obtained data consistent with previous studies.

Lamarao et al. (2012) also pointed that RSV bronchiolitis was more common in first year of life with a rate of $63.1 \%$. Accordingly, in a study carried out by Xiao et al. (2012) 1165 infants had a diagnosis of viral bronchiolitis in whom were mostly between age of six month and one year. Papenburg et al. (2012) revealed that RSV infection was more common and had a more severe course before age of 5 months. The study was conducted in United Kingdom with 1000 children aged < three years. Consistently with previous reports, we investigated that viral LRTI were more frequent in first year than between ages of 12 and 24 months (Lamarão et al., 2012; Xiao et al., 2012; Papenburg et al., 2012). Besides, Lamarao et al. (2012) detected RSV antigens from nasopharyngeal aspirates in $24 \%$ of infants while Xiao et al detected an RSV antigen positivity in $27 \%$ of patients and reported rhinovirus in $17 \%$ of all subjects as the second most common causative viral agent (Xiao et al., 2012). Papenburg et al. (2012) stated that RSV is the most common causative agent of bronchiolitis while human metapneumovirus was the second. Since patients with clinically high suspicion of RSV infection may be tended to be ordered rapid antigen testing more than others, we found a higher RSV positivity (44\%) than previous studies. Hospitalization incidence was $77.2 \%$ and it was attributed hospitalization of infants with severe enough bronchiolitis. When compared to mentioned three studies, RSV positivity in our study was higher than previous studies but, results those based on age and gender were similar.

The peak RSV infection season ranges from November to March in temperate climates. Also, infants born in this season are more likely to be infected with RSV (Khor et al., 2012). In a retrospective study of 27 years performed by Khor et al. (2012) data of 10269 infants with RSV infection were analyzed and it was found to be that RSV infection has a peak incidence in especially last months of the year and infants born in this season are riskier to get infection during first year of life (Khor et al., 2012). The infants born in December and January were more prone to RSV infection than others as stated in our study. Although having siblings who attend nursery or school, smoke exposure, being breastfed for a lesser period and prematurity are known risk factors for RSV bronchiolitis, there was no significant difference between two groups based on these variables (Papenburg et al., 2012).

Smoke exposure, larger families with three or more children at home, prematurity ( $<35$-week gestation), congenital heart diseases are determined as risk factors for severity of RSV infection, extension of hospital stay and oxygen requirement by Papenburg et al. (2012). Including almost hospitalized infants with LRTI rather than healthy subjects as control group, describes the difference between literature and our study. No significant difference was found between two groups for duration of hospitalization and oxygen requirement, and low oxygen saturation (hypoxemia) level.
However, clinical findings of infants with RSV bronchiolitis such as tachypnea and retraction were similar to those with other viral LRTIs. It is difficult to distinguish bronchiolitis and pneumonia caused by viral agents. Tachypnea, intercostal and subcostal retractions along with nasal flaring, wheezing, rhonchi and crackles are common findings. As confirmed in our study, physical examination does not create distinctive features for etiologic agents (Gruber et al., 2002). Clinical variables such as fever, tachypnea, retractions, level of oxygen saturation and need for hospitalization did not differ among groups according to outcomes. Apnea can occur in especially young infants due to RSV infection. Guinea et al. (2007) reported an apnea incidence of $25.7 \%$ in infants age $<$ six week and with severe bronchiolitis among $284 \mathrm{RSV}$ positive subjects. Apnea incidence was 3\% $(n=3)$ in RSV $(+)$ group and $1 \%(n=1)$ in RSV (-) group. Because of a small study group and cases included older than one month, apnea incidence was not significantly different between two groups. Albeit fever is rare or low-grade in RSV bronchiolitis, it is more common and higher in human metapneumovirus or adenovirus bronchiolitis (Tristram,1997). The fever of RSV (+) patients was low-grade with a rate of $37.6 \%$ that was consistent with literature (Tristram et al., 1997).

RSV bronchiolitis may cause recurrent episodes of wheezing during childhood. Patients were followed up for six months after enrollment. No statistically significant difference was found between RSV (+) group and RSV (-) group for wheezing episodes $(28.2 \%$ versus $31.5 \%)$. In a meta-analysis performed by Daniel et al., it was indicated that Human Rhino Virus (HRV) can also cause recurrent wheezing episodes and asthma as RSV, even more may generate a higher risk (20). Our results supported this data. Hence other causative agents have not been screened, we cannot issue an association between other causative agents and recurrent wheezing episodes and asthma. Hospitalization rate was as high as $77.2 \%$ in this study. Therefore, a great proportion of infants received intravenous fluid as expected $(65.9 \%$ of RSV $(+)$ infants and $68.5 \%$ of RSV (-) infants). Avoidance of antibiotic use is offered after detection of viral agents in literature. However rapid tests reduces antibiotic use, antibiotic, systemic steroid, inhaler beta agonist inhaler hypertonic $\mathrm{NaCl}$ and racemic epinephrine use were similar for both groups (Luksic et al., 2013). It was attributed to empirical antibiotic treatment for suspected secondary bacterial infections.

If beneficial, bronchodilator agents like inhaler salbuterol or racemic epinephrine are recommended for severe dyspneic infants caused by RSV bronchiolitis or other viral bronchiolitis (Watts, 2007; Langley et al., 2005). Since almost our patients were hospitalized because of severe enough tachypnea and dyspnea, bronchodilator use was so high $(90.6 \%$ in RSV (+) group and $88 \%$ in RSV (-) group). Zhang et al. (2008) has proven improving effect on edema of small airways and facilitating effect on mucus excretion of nebulized hypertonic saline in a meta-analysis conducted with 1095 patients. In 
addition to being beneficial and safe, it shortens the hospital stay. Because the first choice was bronchodilators and response of patients to bronchodilators were good, we have not administered hypertonic saline as a main treatment method (5.9\% in RSV (+) group and 6.5\% in RV (-) group).

Systemic steroid and inhaler steroid are just used for adults and children with recurrent wheezing episodes. Even if they alleviate bronchial secretions and airway obstruction, metaanalysis have proven no effectiveness on infants with bronchiolitis. Patel et al. (2004) found no significant difference between systemic steroid and placebo in terms of hospitalization, oxygen need and dyspnea through a metaanalysis with data of 1198 infants. Bronchodilator and steroid use were quite common in our clinic but our study has lacking data about prognosis after discharge. Thus we could not arrive at any conclusion about this issue. Inhaler steroid use was higher in RSV (-) patients owing to more frequent recurrent wheezing episodes in this group. Only two patients received palivizumab during hospitalization. None of patients has received antiviral therapy.

During RSV bronchiolitis, WBC count and CRP values are almost in normal limits. By a conducted study with 1050 acute bronchiolitis diagnosed infants, Lamaro et al. (2012) showed that WBC count and CRP were significantly lower RSV bronchiolitis group than other group. Conversely, Bicer et al. (2013) determined high levels of CRP and erythrocyte sedimentation in 301 inpatients aged below 9 with viral LRTI. White blood cell counts were increased in children with adenovirus and rhinovirus associated viral LRTI and marked monocytosis was revealed in adenovirus infection when compared to other viruses including RSV (Bicer et al., 2013). An explanation for non-significant difference for WBC count and CRP between two groups in our study is exclusion of patients with suspected bacterial pneumonia. But as expressed in literature, WBC count and CRP values were high in patients with either RSV bronchiolitis or others. While ALT values were in normal limits, AST values had increased in RSV (+) group and were higher than another group (44\% in RSV $(+)$ group, $24 \%$ in RSV (-) group; $\mathrm{p}=0.003)$. Extrapulmonary involvement in RSV infection is rare. However, it may cause convulsion, hyponatremia, hepatitis and heart failure. Nine patients (5\%) among all subjects developed heart failure during infection course. Eisenhut et al. (2006) detected aminotransferase increase in $49 \%$ of intensive care unit patients who were hospitalized for RSV associated LRTI. The incidence may rise to as high as $80 \%$ in children with concurrent congenital heart disease. AST elevation is consistent with those previously reported in our study. Again, in the same study, hyponatremia was determined in 33\% RSV $(+)$ patients and thought to be result of SIADH. Four children had seizure because of hyponatremia whose simultaneously serum ADH were increased as a result of SIADH (Eisenhut et al., 2006). Likewise, in our study, hyponatremia was more frequent in $\mathrm{RSV}(+)$ patients $(31.7 \%$ of $\mathrm{RSV}(+)$ patients and
$5.8 \%$ of RSV (-) patients; $\mathrm{p}<0.001)$. The possible mechanisms of hyponatremia are SIADH, reduced oral intake and inappropriate fluid replacement (Eisenhut et al., 2006). Since significant increase for urine specific gravity was observed in RSV $(+)$ patients $(\mathrm{p}=0.007)$, hyponatremia was correlated to reduced oral intake instead of SIADH. In total, $49 \%$ of the urine specimens were obtained, so a prediction could not be made about SIADH incidence fort this study. Only one patient had low platelet count. Although no significant difference was found between platelet count and RSV positivity, MPV was significantly lower in RSV (+) patients than RSV (-) patients (71.8\% and $18.9 \%$ respectively). Renshaw et al. (2013) performed bronchoscopy to 158 patients with diagnosis of pneumonia or airway obstruction and compared MPV values between RSV $(+)$ patients and healthy controls. The prediction of viruses was made by viral culture through specimens obtained from bronchoalveolar lavage or rapid tests with immunochromatographic method. They also argued that MPV values were significantly lower in $\mathrm{RSV}(+)$ patients than randomly selected healthy controls and a MPV value $<9.9 \mathrm{fl}$ had a sensitivity of $71 \%$ and specificity of $49 \%$ for RSV (Renshaw et al., 2013). Comparing MPV values of RSV (+) and RSV (-) patients with LRTI from same age and similar clinical and demographic features instead of healthy controls was the superiority of our study. Karadağ et al. (2013) observed decreased MPV values in total of 196 children with community acquired pneumonia, either hospitalized $(n=108)$ or out patients $(n=88)$ when compared to healthy subjects. They suggested a cut-off value of $8.1 \mathrm{fL}$ for MPV. Mete et al. (2014) demonstrated no significant difference for MPV between rota virus gastroenteritis and gastroenteritis caused by other viral agents. Nevertheless, there was significant difference between rotavirus gastroenteritis and healthy controls. MPV is associated with platelet age. Previous studies suggested that increased MPV may be associated with diabetes, obesity and alcohol use while platelet count is normal. Furthermore, decreased MPV value may be associated with various infections and may be used as acute phase reactant as well (Renshaw et al., 2013; Karadag et al., 2013; Mete et al., 2014). Whereas a significant difference for MPV was revealed between RSV positive and negative group, there was no significant difference for CRP and white blood cell. Although the mechanism which RSV causes a decrease in MPV is not clear, it can be used as a parameter in differential diagnosis of RSV and non-RSV bronchiolitis. Further investigations are required to identify this mechanism.

RSV infection is more frequent in first two months of life. Additionally, MCV is physiologically increased in same period. Thus, we suggest that, high MCV values are detected as a consequence of physiologic increase in first two months rather than RSV infection. Besides, MCV is physiologically decreased between eight to 12 weeks because of physiologic anemia (BHL, 1981). Our study has a limitation due to the age group of RSV. Since the physiologic anemia of infancy is 
detected between 8 to 12 weeks, we couldn't evaluate physiologic anemia and anemia related MPV decrease in both RSV (+) and RSV (-) groups. The count of premature and palivizumab received infants were too low to make a suggestion about RSV (+) and (-) infants for hospital stay and oxygen need. Further investigation is needed for palivizumab use in prevention of RSV.

\section{Conflict of interest}

None to declare.

\section{Acknowledgments}

There is no financial or personal relationship which can cause a conflict of interest regarding this article and this research has not been funded by an organization.

\section{References}

1. BHL. Refernece Values in Infancy and Childhood. In: Nathan DG aOF, editor. Hematology of Infancy and Childhood. Philadelphia PA: WB Saunders Co, 1981, 1552-1574.

2. Bicer, S., Giray, T., Çöl, D., Erdağ, G.Ç., Vitrinel, A., 2013. Virological and clinical characterizations of respiratory infections in hospitalized children. Ital. J. Pediatr. 39(22), 1-10.

3. Boyce, T.G., Mellen, B.G., Mitchel, E.F., Wright, P.F., Griffin, M.R., 2000. Rates of hospitalization for respiratory syncytial virus infection among children in Medicaid. J. Pediatr. 137(6), 865-870.

4. Collins, P.L., Chanock, M.K., 1996. Respiratory Syncytial Virus. In: Fields BN KD, Howley PM., editor. Fields Virology. 3rd ed. Philadelphia: Lippincott-Raven Publishers, 1313-1351.

5. Eisenhut, M., 2006. Extrapulmonary manifestations of severe respiratory syncytial virus infection-a systematic review. Crit. Care. 10(4), R107.

6. Geskey, J.M., Cyran, S.E., 2012. Managing the morbidity associated with respiratory viral infections in children with congenital heart disease. Int. J. Pediatr. 646780.

7. Gruber, W.C. Bronchiolitis. Long S.S. PLK, Prober C.G, editor. Principles and Practice of Pediatric Infectious Diseases. 1st ed. Churchill Livingstone, 2002, 246-250.

8. Handforth, J., Friedland, J., Sharland, M., 2000. Basic epidemiology and immunopathology of RSV in children. Paediatr. Respir. Rev. 1(3), 210-214.

9. Jackson, D.J., Lemanske, R.F., 2010. The role of respiratory virus infections in childhood asthma inception. Immunol. Allergy Clin. North. 30(4), 513-522.

10. Karadag-Oncel, E., Ozsurekci, Y., Kara, A., Karahan, S., Cengiz, A.B., Ceyhan, M., 2013. The value of mean platelet volume in the determination of community acquired pneumonia in children. Ital. J. Pediatr. 39, 16.

11. Khor, C.S., Sam, I.C., Hooi, P.S., Quek, K.F., Chan, Y.F., 2012. Epidemiology and seasonality of respiratory viral infections in hospitalized children in Kuala Lumpur, Malaysia: A retrospective study of 27 years. BMC Pediatr. 12(1), 1.

12. Kristensen, K., Stensballe, L.G., Bjerre, J., Roth, D., Fisker, N., 2009. Risk factors for respiratory syncytial virus hospitalisation in children with heart disease. Arch. Dis. Child. 94(10), 785-789.

13. La Vla, W.V., Marks, M.I., Stutman, H.R., 1992. Respiratory syncytial virus puzzle: Clinical features, pathophysiology, treatment, and prevention. J. Pediatr. 121(4), 503-510.
14. Lamarão, L.M., Ramos, F.L., Mello, W.A., Santos, M.C., Barbagelata, L.S., 2012. Prevalence and clinical features of respiratory syncytial virus in children hospitalized for communityacquired pneumonia in northern Brazil. BMC Infect. Dis. 12(1), 1.

15. Langley, J.M., Smith, M.B., LeBlanc, J.C., Joudrey, H., Ojah, C.R., 2005. Racemic epinephrine compared to salbutamol in hospitalized young children with bronchiolitis: A randomized controlled clinical trial. BMC Pediatr. 5(1), 7.

16. López Guinea, A., Casado Flores, J., Martín Sobrino, M.A., Espínola Docio, B., de laCalle Cabrera, T., Serrano, A., 2007. Severe bronchiolitis. Epidemiologyand clinical course of 284 patients. An. Pediatr. 67(2), 116-122.

17. Lukšić, I., Kearns, P.K., Scott, F., Rudan, I., Campbell, H., Nair, H., 2013. Viral etiology of hospitalized acute lower respiratory infections in children under 5 years of age- systematic review and meta-analysis. Croat Med. J. 54(2), 122-134.

18. Mete, E., Akelma, A.Z., Cizmeci, M.N., Bozkaya, D., Kanburoglu, M.K., 2014. Decreased mean platelet volume in children with acute rotavirus gastroenteritis. Platelets. 25(1), 5154.

19. Murray, P.R., Pfaller, M.A., Tenover, F.C., Yolken, R.H., Manuel of Clinical Microbiology. 7th ed. Washington D.C: American Society for Microbiology Press, 1999, 942-958.

20. Navas, L., Wang, E., de Carvalho, V., Robinson, J., Canada, P., 1999. Improved outcome of respiratory syncytial virus infection in a high-risk hospitalized population of Canadian children. J. Pediatr. 121(3), 348-354.

21. Ohuma, E., Okiro, E., Ochola, R., Sande, C., Cane, P., 2012. The natural history of respiratory syncytial virus in a birth cohort: the influence of age and previous infection on reinfection and disease. Am. J. Epidemiol. 176(9), 794-802.

22. Papenburg, J., Hamelin, M.È., Ouhoummane, N., Carbonneau, J., Ouakki, M., 2012. Comparison of risk factors for human metapneumovirus and respiratory syncytial virus disease severity in young children. J. Infect. Dis. 206, 178-189.

23. Patel, H., Platt, R., Lozano, J., Wang, E., 2004. Glucocorticoids for acute viral bronchiolitis in infants and young children. Cochrane Libr. 3.

24. Prober, C.G., Sullender, W.M., 1999. Advances in prevention of respiratory syncytial virus infections. J. Pediatr. 135(5), 546-558.

25. Tristram, D.A., WRC. Respiratory Syncytial Virus. Murray, P.R., Pfaller Mac, B.E., Tenover, F.C., Yolken, R.H., editor. Manual of Clinical Microbiology. Washington: ASM Pres, 1997, 932.

26. Renshaw, A.A., Drago, B., Toraya, N., Gould, E.W., 2013. Respiratory syncytial virus infection is strongly correlated with decreased mean platelet volume. Int. J. Infect. Dis. 17(9), 678-80.

27. Watts, K.D., Wheezing infants: Bronchiolitis. Behrman, R.E., Jenson, H.B., editor. Nelson Textbook of Pediatrics. 18th ed. Philadephia: WB Saunders, 2007, 1773-1777.

28. Walsh, E.E., McConnochie, K.M., Long, C.E., Hall, C.B., 1997. Severity of respiratory syncytial virus infection is related to virus strain. J. Infect. Dis. 175(4), 814-820.

29. Xiao, N., Zhang, B., Duan, Z., Xie, Z., Zhou, Q., 2012. Viral etiology of 1165 hospitalized children with acute lower respiratory tract infection. Chin. J. Contemp. Pediatr. 14(1), 28-23.

30. Zhang, L., Mendoza-Sassi, R.A., Wainwright, C., Klassen, T.P., 2008. Nebulized hypertonic saline solution for acute bronchiolitis in infants. Cochrane Database Syst. Rev. 4. 